\title{
Effect of Rashba splitting on ultrafast carrier dynamics in BiTeI
}

\author{
Anna S. Ketterl, ${ }^{1}$ Beatrice Andres, ${ }^{1}$ Marco Polverigiani, ${ }^{1}$ Vladimir Voroshnin $\odot,{ }^{2,3}$ Cornelius Gahl ${ }^{\circ},{ }^{1}$ Konstantin A. Kokh, ${ }^{4,5}$ \\ Oleg E. Tereshchenko, ${ }^{5,6}$ Evgueni V. Chulkov, ${ }^{2,7,8}$ Alexander Shikin, ${ }^{2}$ and Martin Weinelt (1) ${ }^{1, *}$ \\ ${ }^{1}$ Freie Universität Berlin, Fachbereich Physik, Arnimallee 14, 14195 Berlin, Germany \\ ${ }^{2}$ Saint Petersburg State University, Institute of Physics, Uljanovskaya 1, 198504 Saint Petersburg, Russia \\ ${ }^{3}$ Helmholtz-Zentrum Berlin für Materialien und Energie, Albert Einstein Straße 15, 12489 Berlin, Germany \\ ${ }^{4}$ V. S. Sobolev Institute of Geology and Mineralogy, 630090 Novosibirsk, Russia \\ ${ }^{5}$ Novosibirsk State University, 630090 Novosibirsk, Russia \\ ${ }^{6}$ A. V. Rzhanov Institute of Semiconductor Physics, 630090 Novosibirsk, Russia \\ ${ }^{7}$ Departamento de Física de Materiales UPV/EHU, Centro de Física de Materiales CFM-MPC, \\ and Centro Mixto CSIC-UPV/EHU, 20080 San Sebastián/Donostia, Basque Country, Spain \\ ${ }^{8}$ International Physics Center (DIPC), 20018 San Sebastián/Donostia, Basque Country, Spain
}

(Received 17 September 2020; revised 13 January 2021; accepted 14 January 2021; published 3 February 2021)

\begin{abstract}
Narrow-gap semiconductors with strong spin-orbit coupling such as bismuth tellurohalides have become popular candidates for spintronic applications. But driving spin-polarized photocurrents in these materials with circularly polarized light requires picosecond lifetimes of the photoexcited carriers and low spin-flip scattering rates. In search of these essential ingredients, we conducted an extensive study of the carrier dynamics on the Te-terminated surface of BiTeI, which exhibits a giant Rashba splitting of both surface and bulk states. We observe a complex interplay of surface and bulk dynamics after photoexcitation. Carriers are rapidly rearranged in momentum space by quasielastic phonon and defect scattering, while a phonon bottleneck leads to a slow equilibration between bulk electrons and lattice. The particular band dispersion opens an inelastic decay channel for hot carriers in the form of plasmon excitations, which are immanent to Rashba-split systems. These ultrafast scattering processes effectively redistribute excited carriers in momentum and energy space and thereby inhibit spin-polarized photocurrents.
\end{abstract}

DOI: 10.1103/PhysRevB.103.085406

\section{INTRODUCTION}

Nonmagnetic materials with strong spin-orbit coupling exhibit intriguing fundamental physics $[1,2]$ as well as various possible spintronic applications [3-8]. In these materials, strong spin-orbit coupling mediates spin splitting. Arguably the most direct influence of spin-orbit coupling on the surface electronic structure is the Rashba effect [9-11]. Systems with so called giant Rashba splitting of several dozen meV have been discovered, one of them the layered crystal BiTeI [12-15]. Due to its lack of inversion symmetry in the bulk crystal structure, BiTeI not only exhibits a giant Rashba splitting of its surface states (RSS), but also Rashba-split bulk states $[12,16,17]$. These features promise a high potential of BiTeI for opto-spintronics operating at room temperature [18]. Further essential prerequisites are long-lived optically driven spin currents.

In this work, we directly determine the carrier lifetimes of both electrons and holes and unravel the corresponding elastic and inelastic scattering channels. To this end, we use timeand angle-resolved photoemission spectroscopy (trARPES) to compare electronic structure and carrier dynamics on pristine BiTeI and BiTeI containing 3\% Mn. Mn doping shifts the

*weinelt@physik.fu-berlin.de crossing of the RSS parabolas by $50 \mathrm{meV}$ and we observe a concomitant signature in hole dynamics. We focus on the dynamics in the Rashba states on the Te-terminated surface, which reveals a significant asymmetry between electrons and holes. We find very effective quasielastic scattering between the Rashba-split bulk and surface states and inelastic scattering within the RSS via surface plasmon emission. Both processes are characteristic for Rashba systems and will diminish the spin polarization of photocurrents. We do not observe an asymmetry upon excitation with circular polarized light.

\section{EXPERIMENTAL METHODS}

The BiTeI crystal was obtained from a stoichiometric loading consisting of beforehand synthesized $\mathrm{Bi}_{2} \mathrm{Te}_{3}$ and $\mathrm{BiI}_{3}$. The compounds were sealed in a quartz ampoule under residual pressure of $10^{-3}$ torr, heated to $600^{\circ} \mathrm{C}$, and then solidified by the modified Bridgman method. The resulting ingot $10 \mathrm{~mm}$ in diameter and $40 \mathrm{~mm}$ in length contained a single crystal grain which was then cleaved to smaller samples by a metal blade.

These samples were glued to transferable sample plates and cleaved in situ at room temperature and a pressure of $2 \times 10^{-9}$ mbar and left at these conditions for at least $12 \mathrm{~h}$ in order to achieve aging of the surface. During that time, the I-terminated surface domains saturate with 
(a)
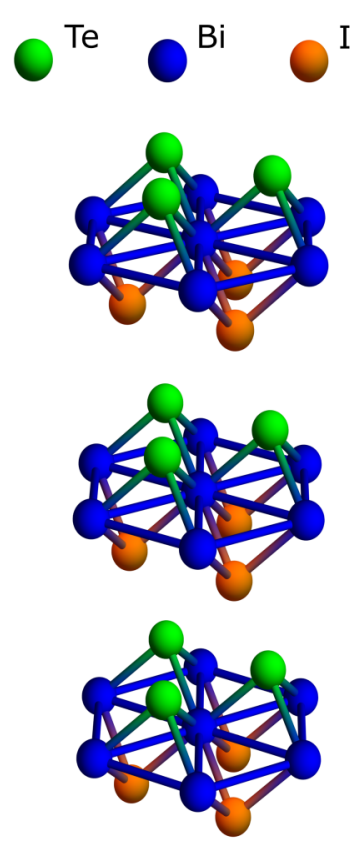

(b)

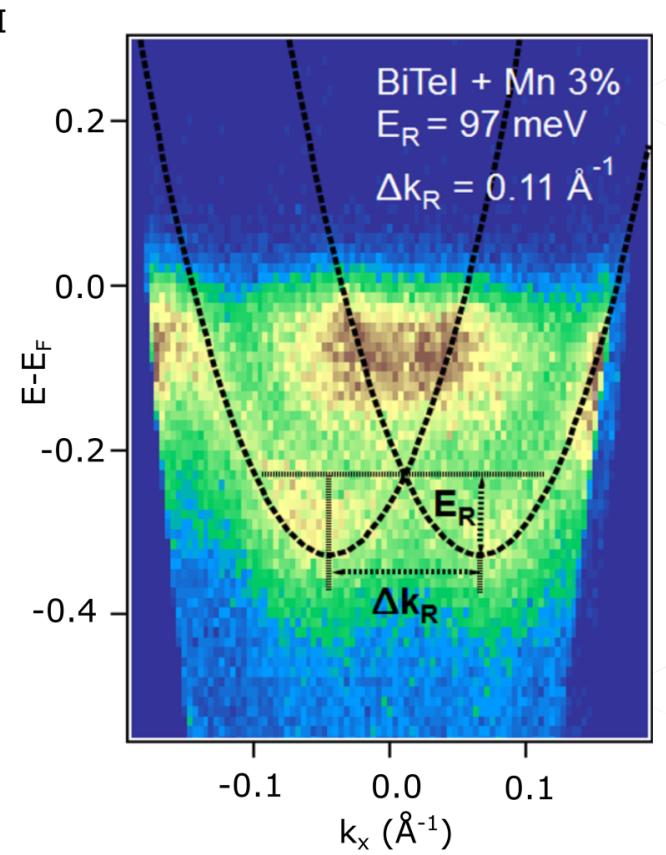

(c)

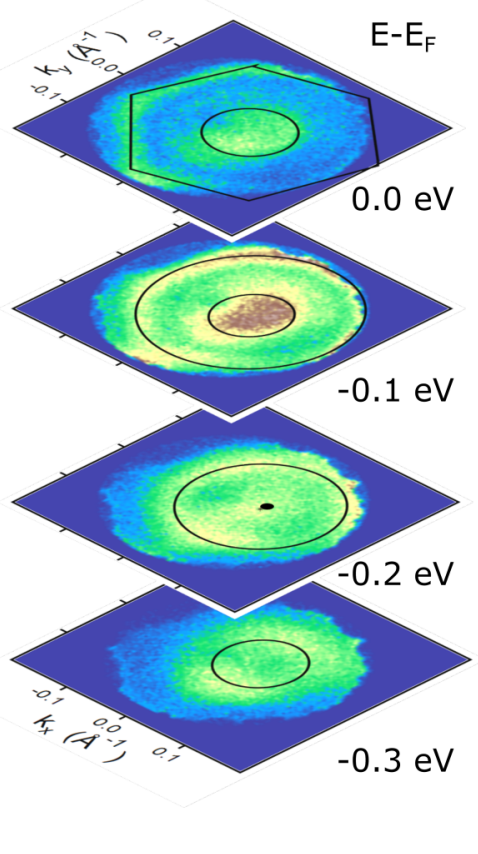

FIG. 1. Crystal and electronic structure of BiTeI. (a) Schematic of the trilayer crystal structure of BiTeI. (b) Dispersion of the RSS of the Te-terminated surface of BiTeI:Mn, recorded along the $k_{x}$ direction at $60 \mathrm{~K}$ sample temperature. The superimposed parabolas were fitted to the dispersion of the RSS. (c) The $k_{\|}$maps at different energies reveal a small hexagonal warping of the RSS at the Fermi energy $E_{F}$, the band bottom of the conduction band at binding energy $E-E_{F}=-0.1 \mathrm{eV}$, overlapping with the inner contour of the RSS, the crossing point of the RSS at $-0.21 \mathrm{eV}$, and the band bottom of the RSS at $-0.31 \mathrm{eV}$.

residual gases, which suppresses the photoemission signal from these domains [19], uncovering the Rashba-split bands of the Te-terminated surface. Throughout the trARPES measurements we observed no further change of the surface. ARPES and trARPES measurements were performed at pressures of $\leqslant 7 \times 10^{-11}$ mbar. During the measurements, the samples were kept either at room temperature or at $60 \mathrm{~K}$. The laser pulses used for ARPES and trARPES were created with a regenerative amplified Ti:Sa oscillator with a repetition rate of $300 \mathrm{kHz}$. The fundamental, with a photon energy of $h v=$ $1.55 \mathrm{eV}$, was used to photoexcite the sample, while its fourth harmonic $(h v=6.2 \mathrm{eV})$ was used for direct photoemission. The two pulses exhibited a cross correlation of 150 fs. Pump and probe pulses were collinear with incidence along $\overline{\Gamma \mathrm{K}}$ at an angle of $45^{\circ}$. Probe pulses are $p$ polarized, pump pulses either $p$ or circularly polarized. Photoelectrons were analyzed by an angle-resolving time-of-flight ( $\mathrm{ToF}$ ) spectrometer (Themis, SPECS $\mathrm{GmbH}$ ), which allowed for simultaneous recording of kinetic energy, and parallel momentum in two dimensions with a polar acceptance angle of $\pm 15^{\circ}$ [20]. As we found no $k_{\|}$ dependence of the dynamics, the presented time-resolved data were averaged over the entire accessible $k_{\|}$space in order to improve the signal-to-noise ratio.

\section{RESULTS}

\section{A. Electronic structure}

BiTeI grows as a layered crystal composed of covalently bound trilayers, stacked by van der Waals forces. The stacking ...-I)-(Te-Bi-I)-(Te-Bi-I)-(Te-... is energetically favorable, leading to the crystal structure depicted in Fig. 1(a) that lacks inversion symmetry. As a consequence, the crystal features charges on its two surfaces, namely a positive/negative partial charge on the Te-/I-terminated surface; these are compensated by corresponding surface band bendings [16]. Stacking faults within the crystal lead to a coexistence of both terminations on the same surface [19]. With domain sizes typically on the order of $100 \mathrm{~nm}[15,19,21]$ and beam diameters in focus of 150-200 $\mu \mathrm{m}$, our laser-based trARPES setup probes in general both domains simultaneously. However, selective aging of the I-terminated surface uncovers the Rashba-split bands of the Te-terminated surface.

Figures 1(b) and 1(c) show 6-eV ARPES spectra obtained on the Te-terminated surface of a Mn-doped sample. We observe the Rashba-split Te-surface bands [highlighted by dashed lines in Fig. 1(b)]. The high blurred intensity at low binding energies, i.e., from the Fermi level to binding energies of $E_{B}=E-E_{F} \sim-0.1 \mathrm{eV}$, stems from the Rashba-split bulk conduction band minimum (CBM). In contrast to photoemission measurements with higher photon energies [12,14,22], we cannot resolve these bands in detail with $h v=6.2 \mathrm{eV}$. This is attributed to the higher quasielastic scattering rates of electrons with low kinetic energies and the larger escape depth that, combined with the surface band bending, leads to a blurring of the bulk bands. The signal from the CBM overlays the inner branches of the Te-RSS, which lie at roughly the same energies and momenta. The cut along the $k_{x}$ direction [Fig. 1(b)] through the crossing point of the RSS was fitted with two parabolas, yielding a Rashba splitting of $\Delta k_{R}=$ $0.11 \pm 0.01 \AA^{-1}$ and a Rashba energy of $E_{R}=97 \pm 5 \mathrm{meV}$. 


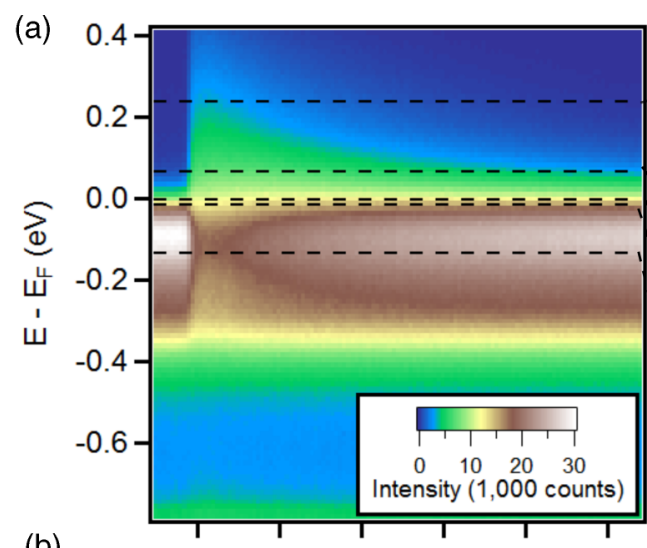

(b)

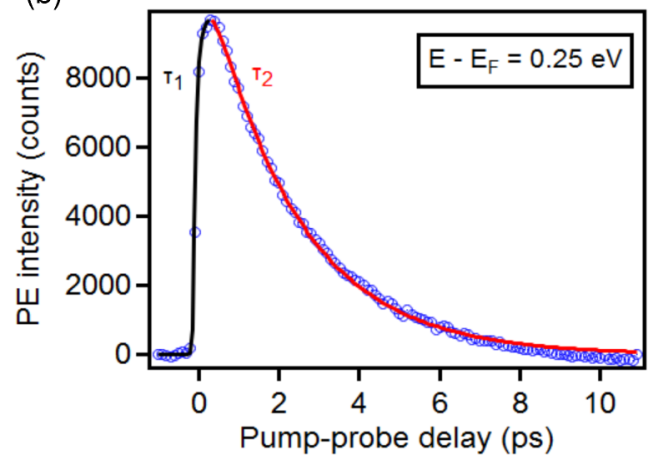

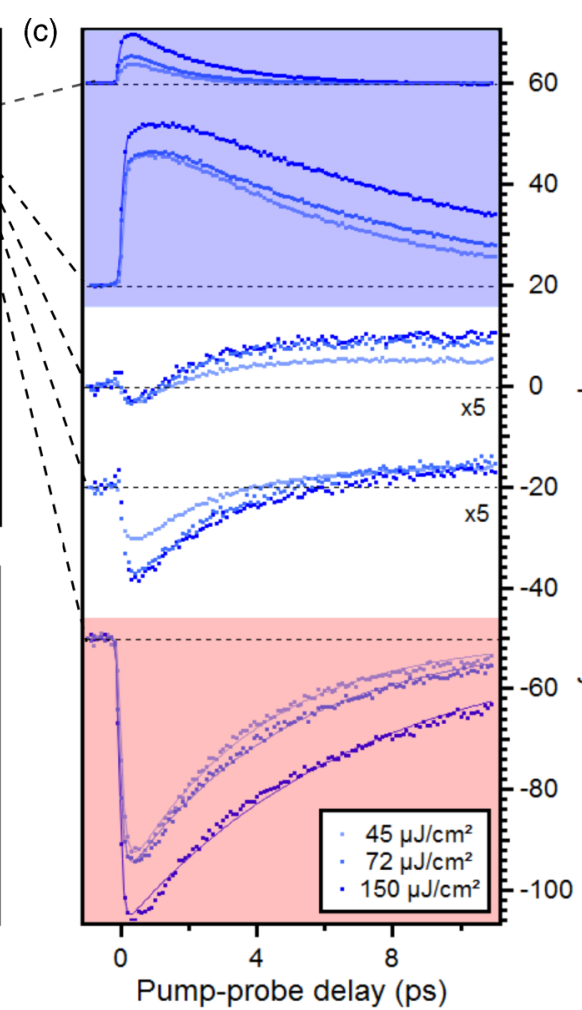

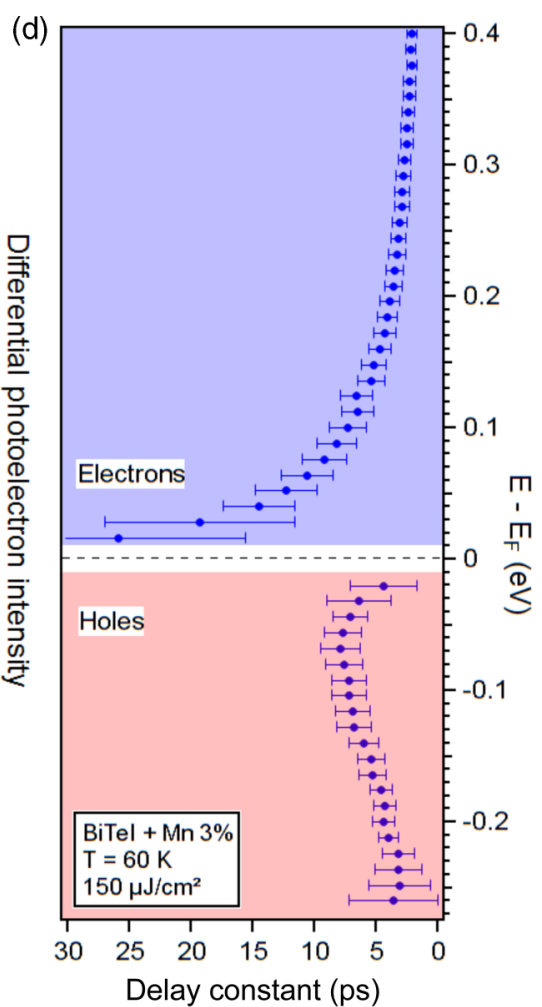

FIG. 2. Ultrafast carrier dynamics under photoexcitation. (a) False-color plot of the photoelectron intensity of the BiTeI:Mn sample, plotted over binding energy and pump-probe delay. The intensity was integrated over the entire accessible $k_{\|}$range. The spectrum was recorded at a temperature of $60 \mathrm{~K}$ with a pump fluence of $150 \mu \mathrm{J} / \mathrm{cm}^{2}$. (b) Exemplary delay spectrum at a single kinetic energy bin $(\Delta E=12 \mathrm{meV})$ at the highest indicated energy in (a), with the corresponding fit of thermalization time constant $\tau_{1}$ and relaxation back to the initial distribution with time constant $\tau_{2}$. (c) Delay spectra and fits recorded with three different pump fluences at the energies indicated by dashed lines in (a). The points represent the measured data, while solid lines are corresponding fits. The traces around the Fermi level have been multiplied by a factor of 5 for clarity. (d) Time constant $\tau_{2}$, obtained from the fitting procedure explained in (b), from the data shown in (a), plotted over the respective energies.

Both of these values are in excellent agreement with previously reported values $[12,14]$. The spectra presented in Fig. 1(c) are obtained by cutting at certain binding energies (one energy bin corresponding to $\Delta E=12 \mathrm{meV}$ ) through the three-dimensional data set. These $\left(k_{x}, k_{y}\right)$ maps reveal the constant-energy contours of the RSS and the CBM. The black lines superimposed in the spectra serve as guide to the eye. The sample was slightly tilted in the $k_{x}$ and $k_{y}$ directions in order to access at least half of the Fermi contour of the outer branch of the RSS and to move the crossing point out of the less sensitive spot at the center of the detector. The outer Fermi contour of the RSS shows weak hexagonal warping, which explains the slight deviation of the RSS parabolas from the fit close to the Fermi edge in Fig. 1(b). The $k_{\|}$range reached in our experiment is restricted by the laser photon energy of $6.2 \mathrm{eV}$, so that the warping at the Fermi energy is only seen in one half of Fig. 1. The weak warping of the Rashba surface state is compatible with that observed in ARPES at higher photon energies (see $\mathrm{SS}_{\mathrm{Te}}$ in Fig. 2(b) of Ref. [14]).

A comparison to measurements on undoped BiTeI reveals that the energetic position and dispersion of the band structure is fairly independent of the Mn doping, save for a small shift of $50 \mathrm{meV}$ in binding energy of the surface state (see Fig. S1 and Table T1 of the Supplemental Material (SM) [23]). This indicates that Mn doping only weakly affects the charge carrier density in BiTeI. However, the RSS parabolas are much better resolved on the Mn-doped sample compared to the spectrum of the pristine sample (not shown). The concomitant higher surface quality is attributed to improved crystal growth upon Mn admixture [12]. Having established the band structure of the investigated surface, we now turn to our time-resolved measurements.

\section{B. Carrier dynamics}

Figure 2(a) shows a false-color plot of the photoemission (PE) intensity over binding energy and pump-probe delay, recorded on the BiTeI:Mn sample at $60 \mathrm{~K}$ and with $150 \mu \mathrm{J} / \mathrm{cm}^{2}$ pump fluence. While the bulk states and the RSS dispersions below the Fermi edge are clearly discernible at all times, the dynamics are independent of either $k_{\|}$direction (see SM [23], Fig. S2). We attribute this to (quasi)elastic phonon and defect scattering [24], effectively redistributing the carriers in $k$ space. Therefore, all presented time-resolved traces are obtained by integrating over the entire accessible $k_{\|}$range. We note that time-resolved ARPES usually probes the population dynamics of excited carriers and not the single photohole lifetime as, e.g., in Ref. [25].

The intensity related to the occupied states decreases during optical excitation, reflecting the gain of signal in the 

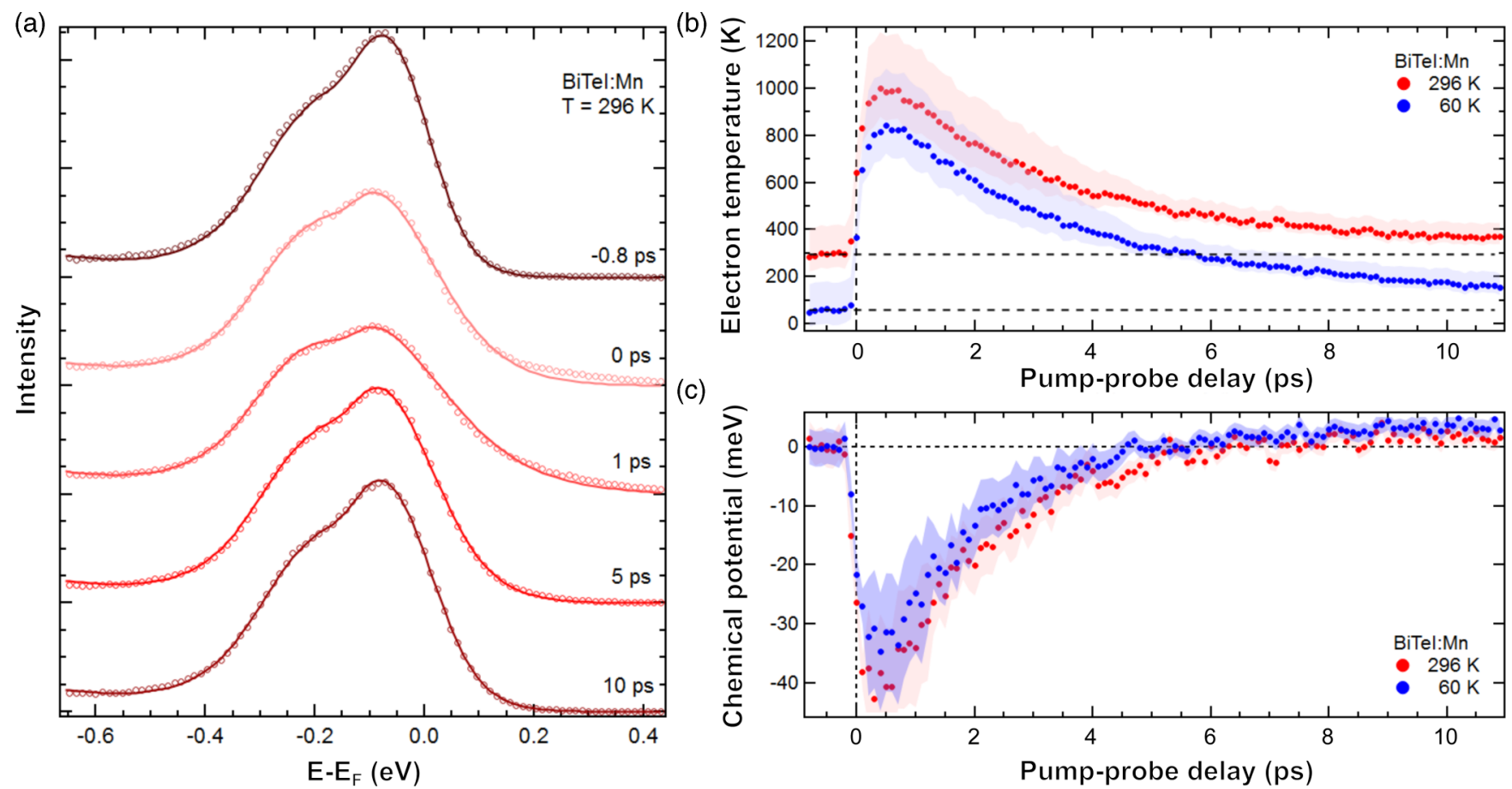

FIG. 3. Evolution of the electronic temperature revealing a phonon bottle neck in electron relaxation. (a) Photoelectron intensity recorded on BiTeI:Mn plotted over the kinetic energy at five characteristic delays integrated over $k_{\|}$. The sample was kept at room temperature and the applied pump fluence was $70 \mu \mathrm{J} / \mathrm{cm}^{2}$. The spectra were fitted with a function comprising surface and bulk model DOS, multiplied with a Fermi function and convolved with a Gaussian to account for experimental resolution. (b) Electronic temperatures over pump-probe delay, extracted from the Fermi distribution in the fits of the DOS, as exemplarily shown in (a). The values have been corrected to fit the initial sample temperatures, as the global fits overestimate the electronic temperature. Light blue and red filled areas indicate error margins. They are not statistical, but denote the regions where reasonable agreement between fit and data can be achieved. (c) Shift of the chemical potential, extracted from the data in the same way as the temperatures displayed in (b).

unoccupied states. Over the next picoseconds, the intensity above $E_{F}$ decays, while the intensity below $E_{F}$ recovers. Figure 2(b) shows an exemplary delay spectrum at $E_{B}=0.25 \mathrm{eV}$ integrated over a single energy bin $(\Delta E=12 \mathrm{meV})$ : We observe a steep change of intensity during the excitation process within the first few hundred femtoseconds $\left(\tau_{1} \sim 200 \mathrm{fs}\right.$, represented by the black part of the curve). The equilibration time $\tau_{1}$ appears to be independent from binding energy, lying in the region of a few hundred femtoseconds for electrons above $E_{F}$ as well as for holes below $E_{F}$ (cf. SM [23], Fig. S3). This confirms thermal equilibration of the electron subsystem by electron-electron scattering within the first $200 \mathrm{fs}$ after excitation which is independent of binding energy [6].

The second timescale $\left[\tau_{2} \sim 5 \mathrm{ps}\right.$; represented by the red part of the curve in Fig. 2(b)] describes the decay of the photoexcited carrier population (here: electrons above $E_{F}$ ). Accordingly, we fit the delay spectra at each energy with a function that consists of two exponentials and a step function, convolved with a Gaussian (FWHM $=150 \mathrm{fs}$ ) to account for the time resolution limited by the cross correlation of pump and probe pulses. Figure 2(c) shows exemplary differential $\mathrm{PE}$ intensities obtained at the energies indicated by the dashed lines in Fig. 2(a), for three different pump fluences, with the corresponding fits. All data are well described by a single exponential decay and we do not observe additional decay components, indicating that edge effects between Te- and I-terminated domains do not affect carrier dynamics. We ex- clude a small energetic region around the Fermi level from the fitting process, which is influenced by the transient shift of the chemical potential during the excitation and recovery (see below).

The time constants $\tau_{2}$ of population decay derived from the data set in Fig. 2(a) are compiled in Fig. 2(d). We can see that indeed $\tau_{2}$, from here on referred to as the decay constant, is in the picosecond range for all recorded energies. It depends on the binding energy of the carriers both above and below the Fermi level, albeit with very different trends: The decay constant of electrons excited above the Fermi level (red shaded area) increases toward the Fermi level in a nonlinear fashion, while the decay constant of the holes (blue shaded area), i.e., the repopulation after the depletion of the occupied bands, only increases approximately linearly toward the Fermi level and drops sharply at low binding energies. These trends generally appear on all samples (see also SM [23], Fig. S4), independently of doping, fluence, and sample temperature. With increasing fluence, the decay constant $\tau_{2}$ universally increases across the entire observed energetic window (see SM [23], Fig. S5).

Most commonly, it is assumed that hot electrons will cool by thermalizing with the lattice via electron-phonon scattering. In order to test how well our data conform with the two-temperature model [26], we extracted the electronic temperatures at each time delay from the data [see Fig. 3(a)]. To that end, we integrated the photoelectron intensity over $k_{\| \mid}$for 
each delay and plotted the resulting experimental fingerprint of the density of states (DOS) over the kinetic energy. This was then fitted with a function representing the electronic twoand three-dimensional DOS (RSS and bulk bands), which was multiplied with a Fermi distribution containing the electronic temperature, and convolved with a Gaussian to account for the experimental resolution (cf. SM [23], Fig. S1).

From Fig. 3(b), we can see that the evolution of the electronic temperature is very reminiscent of the predictions of the two-temperature model. The first exception is the data points within one picosecond after excitation, which show an evolution that could most fittingly be described as "too flat." However, as we have already discussed, these data points are the least reliable, as the electronic temperature is ill defined, as long as the electronic distribution is not fully thermalized yet.

Evidently, heat is dissipated from the electronic system to lattice excitations. However, as the second exception of the two-temperature model we find that even $11 \mathrm{ps}$ after photoexcitation, the electronic system is still at an elevated temperature of $\sim 80 \mathrm{~K}$ above the starting temperature. This is much higher than the expected raise in sample temperature. From a model ansatz with a simple 3D DOS for the CBM (with $m^{*} \sim 0.19 m_{e}$ ) [27], and a phononic specific heat according to the Debye model $\left(\theta_{\mathrm{D}}=87 \mathrm{~K}\right.$ for BiTeI) [27], we obtain a maximal increase of the lattice temperature of $3 \mathrm{~K}$, even at the highest applied fluences (see SM [23]). We therefore conclude that $11 \mathrm{ps}$ after excitation, the electronic system is not yet in equilibrium with the lattice. To prove that the electron temperature $T_{e}$ is not fully recovered at $10 \mathrm{ps}$ it is sufficient to fit the spectrum in Fig. 3(a) at 10 ps delay and compare to the spectrum before pumping at $-0.8 \mathrm{ps}$. To fit both spectra we do not need to shift or broaden the DOS peaks. However, the Fermi distribution is clearly wider at $10 \mathrm{ps}$ delay, yielding an electron temperature of $140 \mathrm{~K}$. The overall decay time of the electron temperature is about $6 \mathrm{ps}$, which is significantly longer than in metals $(<1 \mathrm{ps})$. This is likewise a rough estimate of the decay time of the photoexcited state in BiTeI.

Parallel to the rise of the electron temperature the chemical potential shifts to lower energies by $30-40 \mathrm{meV}$ [see Fig. 3(c)]. This observation reflects the expected temperature dependence of the chemical potential in a DOS with a positive slope. The chemical potential recovers on the ps timescale but slightly overshoots, which indicates that carriers are excited across the band gap from the valence band.

From the PE spectra (cf. Fig. 1(b) and SM [23]), we know that the relative contribution of the surface states to the total intensity is larger below $E_{F}$ than above. At binding energies of more than $-0.15 \mathrm{eV}$, the DOS is even derived purely from the RSS. However, it is hard to predict how the change of dimensionality alone influences, e.g., electron-phonon coupling as well as the screening of electron-electron interactions [28], especially since the coupling between surface and bulk states is unknown. Here we gain more insight from the dependencies of the decay constant $\tau_{2}$ below $E_{F}$.

Unlike the dynamics above $E_{F}$, which show no significant temperature dependence at any energy (cf. SM [23], Fig. S6), the hole dynamics below $E_{F}$ are clearly temperature dependent [cf. Figs. 4(a) and 4(b)]. The trend itself stays the same-a linear increase toward the Fermi level with a sharp drop at very low binding energies-but the slope is significantly flatter at room temperature, leading to an almost energy-independent $\tau_{2}$ at binding energies above $50 \mathrm{meV}$.

Having established the first requirement for the generation of spin-polarized currents in BiTeI, namely a long-lived electron and hole population, a second requirement is the coupling of the photon angular momentum to the electron spin, as proposed for both topological insulators and Rashba materials $[5,6,18,29]$. This type of coupling would lead to asymmetric excitation patterns, which are observable in pump-probe experiments [20,30,31]. To investigate this possibility on BiTeI, we irradiated the sample with circularly polarized light of $1.5 \mathrm{eV}$ photon energy, carefully adjusting the circular polarization (cp) at the sample position by use of a Soleil-Babinet compensator. By adding spectra recorded at zero delay with right $\mathrm{cp}$ and left cp pump polarization, respectively, we obtain spectra with very well discernible excitation of the RSS and CBM [cf. Fig. 5(a)]. Subtracting the respective spectra and dividing by the sum yields the pump-induced circular dichroism in the angular distribution (CDAD) spectra presented in Figs. 5(b) and 5(c). From these data it is evident that within the 150 -fs cross correlation of the laser pulses no polarizationdependent excitation takes place.

\section{DISCUSSION}

Our 6-eV ARPES measurements agree with previously reported photoemission studies of the BiTeI band structure. Manganese doping slightly increases the binding energy of the surface state and leads to a better observability of the former by improving the crystallinity of the sample.

Time-resolved ARPES measurements show that the carrier dynamics after photoexcitation with $1.5 \mathrm{eV}$ photon energy are very complex, due to the different involved subsystems, i.e., the two-dimensional RSS and the three-dimensional CBM. Both above and below the Fermi level, we observe a fast thermalization of the photoexcited hot electron gas, on a timescale of $200 \mathrm{fs}$. As we see no $k$ dependence of the dynamics either above or below the Fermi level, we conclude that phonon and defect scattering redistribute momentum on even shorter timescales.

\section{A. Electron dynamics}

The electron dynamics, i.e., the dynamics above $E_{\mathrm{F}}$, are mainly governed by bulk states. Energy dissipation from the electronic system after excitation can be described with electron-phonon scattering. However, we observe a slow equilibration between electrons and the lattice, which is not completed $11 \mathrm{ps}$ after excitation. We explain this behavior with a phonon bottleneck, as also observed, e.g., on graphene $[32,33]$. The six optical phonon branches of BiTeI are found in the energy range of 7 to $18 \mathrm{meV}$, separated from the three acoustic branches below $6 \mathrm{meV}$ [34]. The electronic system very quickly thermalizes with the subsystem of optical phonons, effectively excited by Fröhlich interaction [Coulomb interaction with the (BiTe) $)^{+} \mathrm{I}^{-}$ions, diverging $\sim 1 / q$, where $q$ is the phonon wave vector] on a timescale on the same order of magnitude as electron-electron scattering. Meanwhile, 


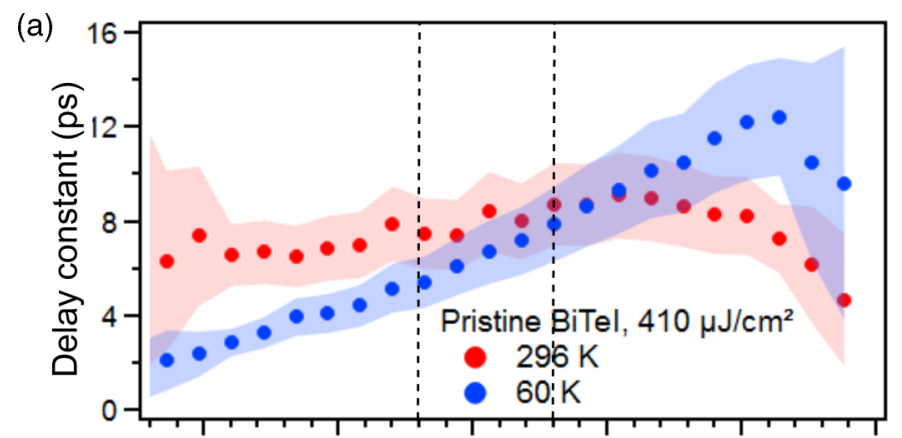

(c)

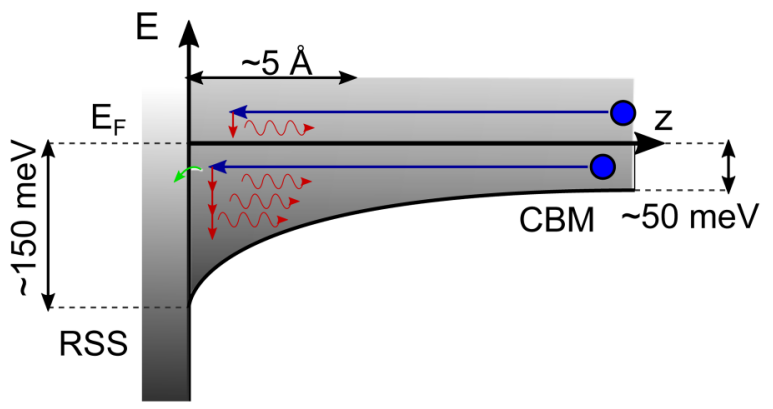

(b)

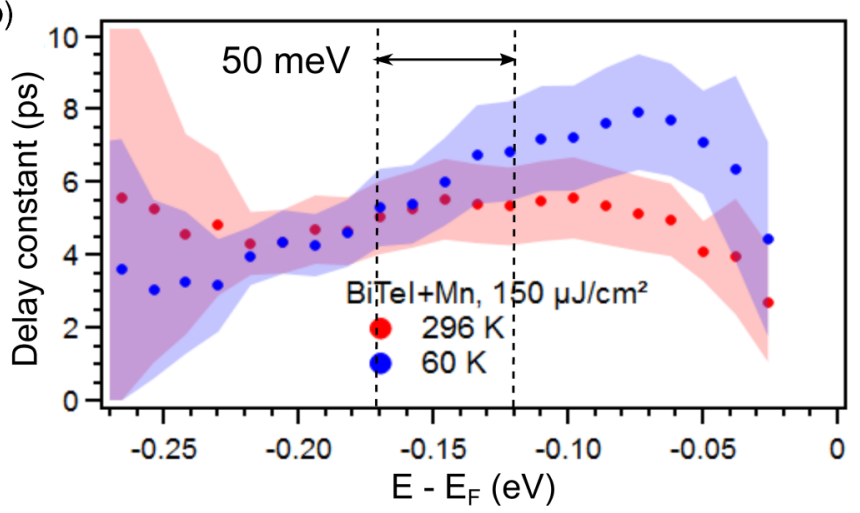

(d)

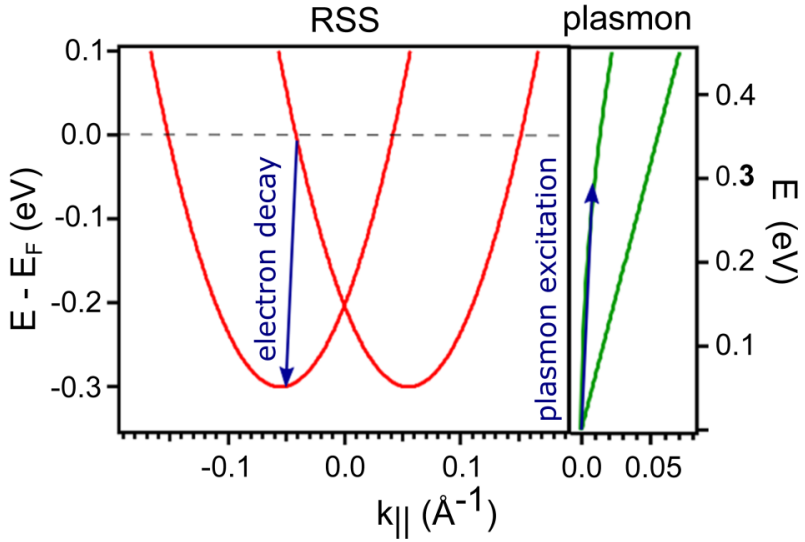

FIG. 4. Temperature dependence of the hole dynamics revealing inelastic electron decay via plasmon excitation. (a) Decay constant $\tau_{2}$ of the carriers below the Fermi level in pristine (a) BiTeI and (b) BiTeI $+3 \% \mathrm{Mn}$. The decay constants were obtained from the fitting procedure explained in Fig. 2 of two different data sets measured on the same samples, but at different temperatures. The pump fluence was 410 and 150 $\mu \mathrm{J} / \mathrm{cm}^{2}$, respectively. Light blue and red filled areas indicate error margins. They are not statistical, but denote the regions where reasonable agreement between fit and data can be achieved. (c) Energy scheme of the band bending on the Te-terminated surface of BiTeI. (d) Schematic of the dispersion of the RSS (red) and surface plasmon dispersion (green). The blue arrows indicate the possible inelastic electron decay channel by plasmon emission.

deformation potential scattering with the acoustic phonon population and equilibration within the phononic system are much less effective leading to elevated electronic temperatures even $11 \mathrm{ps}$ after photoexcitation. Even though scattering with acoustic phonons has been found to be very effective in BiTeI in the transport regime at meV excitation energies [27,35,36], it is less effective for the optically excited hot electron gas that is created by the pump pulse. In this case, long relaxation times of the optical phonons with the remaining phonon population significantly slows down the dissipation of heat from the electronic system [37,38].

\section{B. Hole dynamics}

Right below the Fermi level, both at $60 \mathrm{~K}$ and room temperature (RT), we observe a sudden decrease of the hole decay times. This kink can be explained with the downward band bending on the Te-terminated surface, that compensates the partial positive surface charge [14]. The static field associated with the band bending remains, giving rise to a field current of electrons toward the surface [cf. Fig. 4(c)]. Or, seen vice versa, the static field facilitates the diffusion of photoholes into the bulk. However, this channel is open only for photoholes in the bulk states, i.e., the CBM, but not for the photoholes in the two-dimensional RSS, and therefore only influences the dynamics close to the Fermi level.

We can estimate the timescale on which these drift currents take place, by making some minor assumptions about the material. From measurements of the conductivity and Hall voltage, we get a lower bound for the mobility of carriers in BiTeI on the order of $2 \times 10^{-2} \mathrm{~m}^{2} / \mathrm{V}$ s at RT and $5 \times 10^{-2}$ $\mathrm{m}^{2} / \mathrm{V} \mathrm{s}$ at $77 \mathrm{~K}[36,39]$. These values are valid for movement of the electrons within the trilayer. The conductivity along the stacking axis is half as large as within the planes [35]. Following Eremeev et al. [16], the difference in potential on the clean Te-terminated surface is approximately $0.23 \mathrm{eV}$ over a depth $d$ of roughly $10 \AA$. However, the bottom of the CBM is situated at approximately $E_{\mathrm{CBM}}=-0.15 \mathrm{eV}$ binding energy. From typical carrier densities [27,36,39] and $m^{*}=0.19 m_{e}$ [27], we obtain $E_{\mathrm{CBM}}-E_{F}=-0.07 \mathrm{eV}$, coinciding with the position of the kink in Fig. 4(b). Therefore, the remaining potential difference is on the order of $0.08 \mathrm{eV}$. If we insert these values, we gain velocities on the order of $v=\mu \mathcal{E} \approx 10^{6} \mathrm{~m} / \mathrm{s}$. This means that the holes would cover the $10 \AA$ (comparable with the Debye length from the space charge region; see also Ref. [16]) of the band bending within a femtosecond, indicating ballistic transport. In fact, we obtain comparable velocities simply solving $E_{k i n}=0.5 m_{e} v^{2}=e \mathcal{E} d$. Once arrived at the surface, the electrons can only decay via phonon emission. The combination of ballistic transport and phonon decay explains the observed decrease of the hole decay constant toward $E_{F}$.

We now turn to the temperature dependence of the photohole decay times, which reveal surface plasmon emission. At first glance surprising, we observe the following carrier dynamics below $E_{F}$ : At RT the relaxation close to the RSS 

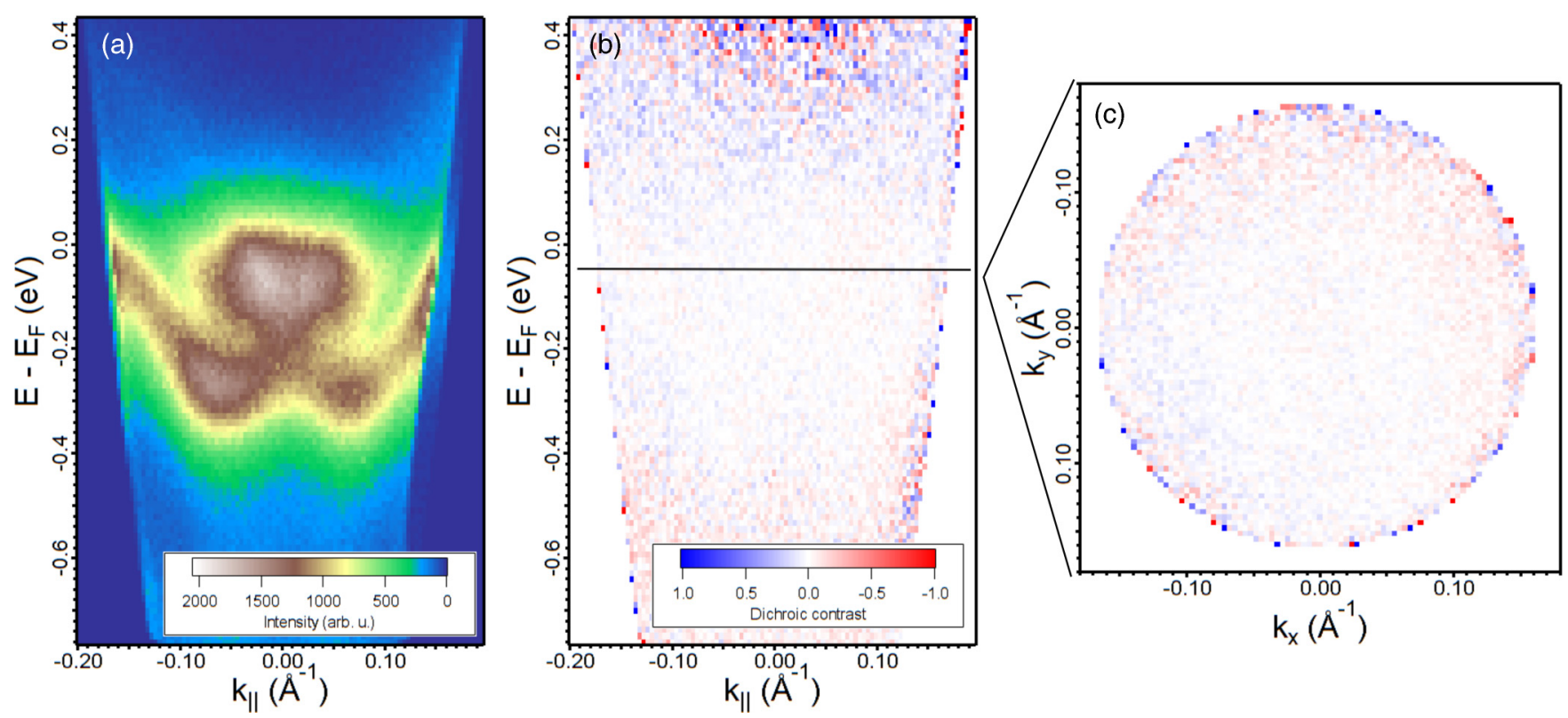

FIG. 5. Missing pump-induced circular dichroism in the angular distribution (CDAD) of photoelectrons indicating the lack of photocurrents. Time-resolved photoelectron spectra recorded with right and left circularly polarized (rcp and lcp) pump pulses of $1.5 \mathrm{eV}$ photon energy and $6.2 \mathrm{eV}$ p-polarized probe pulses at zero delay on a BiTeI:Mn sample at $60 \mathrm{~K}$. (a) Sum of spectra recorded with rcp and lcp pump polarization, respectively. The RSS and conduction band are quite discernible. (b), (c) Difference spectra, i.e., pump-induced CDAD patterns, for two different cuts through the $3 \mathrm{D}$ data set. No dichroic signal emerges, when the polarization is carefully adjusted at the sample position.

band bottom is slower, while the relaxation closer to the Fermi level is faster than at a sample temperature of $60 \mathrm{~K}$ (see Fig. 4). This leads to a crossing point of the two trends, which lies at approximately $-120 \mathrm{meV}$ binding energy for the pristine sample and at approximately $-170 \mathrm{meV}$ binding energy for the Mn-doped sample. Even though for both temperatures the slopes and offsets of $\tau_{2}$ change with the fluence, the crossing point of the two temperature trends stays in the same region independently of excitation fluence for both samples. The peculiar temperature dependence of the dynamics indicates that there is a decay channel which is only effective at low temperatures. This decay channel has to redistribute electrons from the CBM or the upper branches of the RSS to the bottom of the RSS, in order to reduce the lifetimes of holes at high binding energies and prolong those at low binding energies. The plausible candidate for such a channel is the surface plasmon proposed by Nechaev et al. [16,40-42]. Normally, the steep dispersion of surface plasmons makes it impossible for surface electrons to couple to plasmon excitations, as small momentum and large energy transfer required to generate the plasmon cannot be simultaneously balanced by inelastic electron decay. However, the Rashba splitting of the parabolic bands allows for a decay process of electrons with large energy but hardly any momentum transfer, namely the decay from the inner branch above the crossing point to the band bottom below the crossing point [cf. blue arrows in Fig. 4(d)]. Figure 4(d) illustrates the dispersion of the RSS (left panel) and of the surface acoustic plasmon [43] and the plasmon of a $2 \mathrm{~d}$ electron gas [44] (right panel). The two blue arrows indicate interband electron decay via plasmon emission, conserving energy and momentum. Electron decay from the upper to the lower RSS branch entails that the crossing point of the two $\tau_{2}$ trends for 60 and $300 \mathrm{~K}$ in the pristine [Fig. 4(a)] and Mn-doped [Fig. 4(b)] sample must coincide with the crossing point of the RSS. This is supported by our data: The band bottom of the RSS on both samples shows a difference in binding energy of roughly $0.05 \mathrm{eV}$, with the RSS of the Mn-doped sample situated at higher binding energies (cf. SM [23], Fig. S1). This is in line with the shift of the crossing point of the decay constants in Fig. 4(b) and corroborates our conclusion that electrons scatter from the upper to the lower RSS branch via plasmon emission. A closing of the plasmon decay channel at RT, due to increased scattering with optical phonons $\left(1 / \tau \propto 2 n_{\mathrm{ph}}+1\right)$ and a concomitant dephasing and broadening of the surface plasmon, will lead to a flattening of the energy dependence of the decay constants. As electronphonon scattering would lead to an increase of the lifetimes toward the Fermi level, even at RT, mirroring the trend of the electrons, we can conclude that surface recombination is the major decay channel for the holes in the RSS. Therefore, an increased surface recombination rate at room temperature via electron-phonon scattering will speed up filling of the upper RSS branches out of the CBM $\left[E-E_{F}=0\right.$ to $-0.1 \mathrm{eV}$; Fig. 1(a)].

The inelastic plasmon decay channel is only open for carriers in the inner branches of the surface state, but not for the outer branches. This should, in turn, lead to $k_{\|}$-dependent dynamics. Above the crossing point, holes in the inner branch live longer than in the outer branch, whereas hole lifetimes are reversed below the crossing point. The lack of $k_{\|}$dependence of the dynamics in our data (cf. SM [23], Fig. S2) can be explained by quasielastic phonon and defect scattering, 
which effectively redistributes carriers in momentum space. However, the 50-meV shift of the crossing point of the decay constants comparing Figs. 4(a) and 4(b) reflects the shift of the crossing of the Rashba parabolas comparing BiTeI and Mn-doped BiTeI, respectively (see Fig. S1 and Table T1 of the SM [23]). This is experimental evidence for interband decay where plasmon emission conserves momentum and energy [see Fig. 4(d)]. Jointly, elastic and inelastic scattering processes shuffle photoexcited carriers horizontally and vertically in between the Rashba spilt bands. Only the "vertical" dependence of the dynamics on the energy (below and above the crossing point) remains, while the $k$ dependence is obscured by ultrafast quasielastic scattering.

Due to the phonon bottleneck the population decay of photoexcited electrons exceeds the 10 picosecond range and may thus support spin-polarized photocurrents [7]. However, since only true backscattering is forbidden by the opposite spin orientations, the observed scattering channels diminish spin polarization already during laser excitation. As we do not observe any spin dependence even during the excitation with circularly polarized light, further investigation of the origin of already observed helicity-dependent photocurrents in BiTeI with even shorter laser pulses seems essential [18].

\section{CONCLUSION}

In summary, the dynamics of the unoccupied states are dominated by bulk electron-phonon interactions, particularly a phonon bottleneck leading to very long equilibration times between the hot electronic system and the lattice.

Furthermore, ballistic transport of bulk carriers to the surface in the residual electrostatic field of the positively charged surface leads to an accumulation of carriers around the Fermi level, where they can decay by surface recombination and electron-phonon scattering.
The hole dynamics at larger binding energies show a significant influence of the particular dispersion of the Rashba-split surface state. At $60 \mathrm{~K}$ sample temperature, the dynamics in the RSS are governed by the coupling of the electronic system to the surface plasmon, leading to a decrease of the decay times toward the band bottom and an increase toward the Fermi level. At RT, we observe that the decay is almost independent of binding energy and therefore dominated by effective quasielastic scattering and surface recombination.

Our data show that quasielastic and inelastic scattering are very effective in redistributing carriers in the Rashba split bands. This is a general observation, which will hold in conducting systems with Rashba-split surface and bulk states. Momentum scattering and plasmon emission lead to a rapid redistribution of carriers diminishing a possible optically induced spin polarization. Thus scattering of carriers with optical phonons and defects has to be reduced to warrant the potential of Rashba materials in opto-spintronic applications.

\section{ACKNOWLEDGMENTS}

This project was supported by the German Research Foundation via the Collaborative Research Center TRR 227 on Ultrafast Spin Dynamics, project A1, and the German-Russian Interdisciplinary Science Center (GRISC). A.S.K. is indebted to the International Max Planck Research School Functional Interfaces in Physics and Chemistry for financial support. B.A. and V.V. acknowledge travel support by GRISC. O.E.T. and K.A.K. were supported by the Russian Science Foundation (Project No. 17-12-01047) and a state contract of ISP and IGM SB RAS. A.S. acknowledges support by Russian Science Foundation (Grant No. 18-12-00062). A.S. and E.V.C. acknowledge support by the Saint Petersburg State University (Grant No. ID 73028629).
[1] M. Z. Hasan and C. L. Kane, Colloquium: Topological insulators, Rev. Mod. Phys. 82, 3045 (2010).

[2] A. Bansil, H. Lin, and T. Das, Colloquium: Topological band theory, Rev. Mod. Phys. 88, 021004 (2016).

[3] S. Datta and B. Das, Electronic analog of the electro-optic modulator, Appl. Phys. Lett. 56, 665 (1990).

[4] M. Studer, G. Salis, K. Ensslin, D. C. Driscoll, and A. C. Gossard, Gate-Controlled Spin-Orbit Interaction in a Parabolic GaAs/AlGaAs Quantum Well, Phys. Rev. Lett. 103, 027201 (2009).

[5] J. W. McIver, D. Hsieh, H. Steinberg, P. Jarillo-Herrero, and N. Gedik, Control over topological insulator photocurrents with light polarization, Nat. Nanotechnol. 7, 96 (2011).

[6] J. Mauchain, Y. Ohtsubo, M. Hajlaoui, E. Papalazarou, M. Marsi, A. Taleb-Ibrahimi, J. Faure, K. A. Kokh, O. E. Tereshchenko, S. V. Eremeev, E. V. Chulkov, and L. Perfetti, Circular Dichroism and Superdiffusive Transport at the Surface of BiTeI, Phys. Rev. Lett. 111, 126603 (2013).

[7] C. Kastl, C. Karnetzky, H. Karl, and A. W. Holleitner, Ultrafast helicity control of surface currents in topological insulators with near-unity fidelity, Nat. Commun. 6, 6617 (2015).
[8] V. V. Volobuev, P. S. Mandal, M. Galicka, O. Caha, J. SánchezBarriga, D. D. Sante, A. Varykhalov, A. Khiar, S. Picozzi, G. Bauer, P. Kacman, R. Buczko, O. Rader, and G. Springholz, Giant Rashba splitting in $\mathrm{Pb}_{1-x} \mathrm{Sn}_{x} \mathrm{Te}$ (111) topological crystalline insulator films controlled by Bi doping in the bulk, Adv. Mater. 29, 1604185 (2017).

[9] E. I. Rashba, Properties of semiconductors with an extremum loop. 1. Cyclotron and combinational resonance in a magnetic field perpendicular to the plane of the loop, Sov. Phys. Solid State 2, 1109 (1960).

[10] Y. A. Bychkov and E. I. Rashba, Properties of a 2D electron gas with lifted spectral degeneracy, Pis'ma Zh. Eksp. Teor. Fiz. 39, 66 (1984) [JETP Lett. 39, 78 (1984)].

[11] Yu. A. Bychkov and E. I. Rashba, Oscillatory effects and the magnetic susceptibility of carriers in inversion layers, J. Phys. C: Solid State Phys. 17, 6039 (1984).

[12] K. Ishizaka, M. S. Bahramy, H. Murakawa, M. Sakano, T. Shimojima, T. Sonobe, K. Koizumi, S. Shin, H. Miyahara, A. Kimura, K. Miyamoto, T. Okuda, H. Namatame, M. Taniguchi, R. Arita, N. Nagaosa, K. Kobayashi, Y. Murakami, R. Kumai, Y. Kaneko, Y. Onose, and Y. Tokura, Giant 
Rashba-type spin splitting in bulk BiTeI, Nat. Mater. 10, 521 (2011).

[13] S. V. Eremeev, I. A. Nechaev, Yu. M. Koroteev, P. M. Echenique, and E. V. Chulkov, Ideal Two-Dimensional Electron Systems with a Giant Rashba-Type Spin Splitting in Real Materials: Surfaces of Bismuth Tellurohalides, Phys. Rev. Lett. 108, 246802 (2012).

[14] A. Crepaldi, L. Moreschini, G. Autès, C. Tournier-Colletta, S. Moser, N. Virk, H. Berger, Ph. Bugnon, Y. J. Chang, K. Kern, A. Bostwick, E. Rotenberg, O. V. Yazyev, and M. Grioni, Giant Ambipolar Rashba Effect in the Semiconductor BiTeI, Phys. Rev. Lett. 109, 096803 (2012).

[15] S. Fiedler, T. Bathon, S. V. Eremeev, O. E. Tereshchenko, K. A. Kokh, E. V. Chulkov, P. Sessi, H. Bentmann, M. Bode, and F. Reinert, Termination-dependent surface properties in the giantRashba semiconductors BiTe $X(X=\mathrm{Cl}, \mathrm{Br}, \mathrm{I})$, Phys. Rev. B 92, 235430 (2015).

[16] S. V. Eremeev, I. A. Nechaev, and E. V. Chulkov, Giant Rashbatype spin splitting at polar surfaces of BiTeI, JETP Lett. 96, 437 (2012).

[17] G. Landolt, S. V. Eremeev, Y. M. Koroteev, B. Slomski, S. Muff, T. Neupert, M. Kobayashi, V. N. Strocov, T. Schmitt, Z. S. Aliev, M. B. Babanly, I. R. Amiraslanov, E. V. Chulkov, J. Osterwalder, and J. H. Dil, Disentanglement of Surface and Bulk Rashba Spin Splittings in Noncentrosymmetric BiTeI, Phys. Rev. Lett. 109, 116403 (2012).

[18] N. Ogawa, M. S. Bahramy, Y. Kaneko, and Y. Tokura, Photocontrol of Dirac electrons in a bulk Rashba semiconductor, Phys. Rev. B 90, 125122 (2014).

[19] S. Fiedler, L. El-Kareh, S. V Eremeev, O. E Tereshchenko, C. Seibel, P. Lutz, K. A Kokh, E. V Chulkov, T. V Kuznetsova, V. I Grebennikov, H. Bentmann, M. Bode, and F. Reinert, Defect and structural imperfection effects on the electronic properties of BiTeI surfaces, New J. Phys. 16, 075013 (2014).

[20] A. S. Ketterl, S. Otto, M. Bastian, B. Andres, C. Gahl, J. Minár, H. Ebert, J. Braun, O. E. Tereshchenko, K. A. Kokh, Th. Fauster, and M. Weinelt, Origin of spin-polarized photocurrents in the topological surface states of $\mathrm{Bi}_{2} \mathrm{Se}_{3}$, Phys. Rev. B 98, 155406 (2018).

[21] C. J. Butler, H.-H. Yang, J.-Y. Hong, S.-H. Hsu, R. Sankar, C.-I. Lu, H.-Y. Lu, K.-H. O. Yang, H.-W. Shiu, C.-H. Chen, C.-C. Kaun, G.-J. Shu, F.-C. Chou, and M.-T. Lin, Mapping polarization induced surface band bending on the Rashba semiconductor BiTeI, Nat. Commun. 5, 4066 (2014).

[22] J. S. Lee, G. A. H. Schober, M. S. Bahramy, H. Murakawa, Y. Onose, R. Arita, N. Nagaosa, and Y. Tokura, Optical Response of Relativistic Electrons in the Polar BiTeI Semiconductor, Phys. Rev. Lett. 107, 117401 (2011).

[23] See Supplemental Material at http://link.aps.org/supplemental/ 10.1103/PhysRevB.103.085406 for further details of the density of states, proof of $k_{\|}$-independent dynamics, electron and hole thermalization times, carrier dynamics in pristine and $\mathrm{Mn}-$ doped BiTeI, and a comparison of electron dynamics at 60 and 296 K.

[24] Th. Fauster, M. Weinelt, and U. Höfer, Quasi-elastic scattering of electrons in image-potential states, Prog. Surf. Sci. 82, 224 (2007).

[25] J. Sánchez-Barriga, M. R. Scholz, E. Golias, E. Rienks, D. Marchenko, A. Varykhalov, L. V. Yashina, and O. Rader, Anisotropic effect of warping on the lifetime broadening of topological surface states in angle-resolved photoemission from $\mathrm{Bi}_{2} \mathrm{Te}_{3}$, Phys. Rev. B 90, 195413 (2014).

[26] S. I. Anisimov, B. L. Kapelovich, and T. L. Perelman, Electron emission from metal surfaces exposed to ultrashort laser pulses, Zh. Eksp. Teor. Fiz. 66, 776 (1974) [JETP 39, 375 (1974)].

[27] L. Wu, J. Yang, M. Chi, S. Wang, P. Wei, W. Zhang, L. Chen, and J. Yang, Enhanced thermoelectric performance in $\mathrm{Cu}$-intercalated BiTeI by compensation weakening induced mobility improvement, Sci. Rep. 5, 14319 (2015).

[28] P. M. Echenique, R. Berndt, E. V. Chulkov, Th. Fauster, A. Goldmann, and U. Höfer, Decay of electronic excitations at metal surfaces, Surf. Sci. Rep. 52, 219 (2004).

[29] P. Hosur, Circular photogalvanic effect on topological insulator surfaces: Berry-curvature-dependent response, Phys. Rev. B 83, 035309 (2011).

[30] H. Soifer, A. Gauthier, A. F. Kemper, C. R. Rotundu, S.-L. Yang, H. Xiong, D. Lu, M. Hashimoto, P. S. Kirchmann, J. A. Sobota, and Z.-X. Shen, Band-Resolved Imaging of Photocurrent in a Topological Insulator, Phys. Rev. Lett. 122, 167401 (2019).

[31] K. Kuroda, J. Reimann, J. Güdde, and U. Höfer, Generation of Transient Photocurrents in the Topological Surface State of $\mathrm{Sb}_{2} \mathrm{Te}_{3}$ by Direct Optical Excitation with Midinfrared Pulses, Phys. Rev. Lett. 116, 076801 (2016).

[32] J. C. Johannsen, S. Ulstrup, F. Cilento, A. Crepaldi, M. Zacchigna, C. Cacho, I. C. E. Turcu, E. Springate, F. Fromm, C. Raidel, T. Seyller, F. Parmigiani, M. Grioni, and P. Hofmann, Direct View of Hot Carrier Dynamics in Graphene, Phys. Rev. Lett. 111, 027403 (2013).

[33] M. J. Stern, L. P. Rene de Cotret, M. R. Otto, R. P. Chatelain, J.-P. Boisvert, M. Sutton, and B. J. Siwick, Mapping momentum-dependent electron-phonon coupling and nonequilibrium phonon dynamics with ultrafast electron diffuse scattering, Phys. Rev. B 97, 165416 (2018).

[34] I. Yu. Sklyadneva, R. Heid, K.-P. Bohnen, V. Chis, V. A. Volodin, K. A. Kokh, O. E. Tereshchenko, P. M. Echenique, and E. V. Chulkov, Lattice dynamics of bismuth tellurohalides, Phys. Rev. B 86, 094302 (2012).

[35] J. Horák, L. Tichý, and P. Lošták, Electrical conductivity of BiTeI crystals, Phys. Status Solidi A 63, 407 (1981).

[36] N. Dich, P. Lošták, and J. Horák, Preparation and basic physical properties of BiTeI single crystals, Czech. J. Phys. 28, 1297 (1978).

[37] M. Scheuch, T. Kampfrath, M. Wolf, K. von Volkmann, C. Frischkorn, and L. Perfetti, Temperature dependence of ultrafast phonon dynamics in graphite, Appl. Phys. Lett. 99, 211908 (2011).

[38] L. Perfetti, P. A. Loukakos, M. Lisowski, U. Bovensiepen, H. Eisaki, and M. Wolf, Ultrafast Electron Relaxation in Superconducting $\mathrm{Bi}_{2} \mathrm{Sr}_{2} \mathrm{CaCu}_{2} \mathrm{O}_{8+\delta}$ by Time-Resolved Photoelectron Spectroscopy, Phys. Rev. Lett. 99, 197001 (2007).

[39] V. A. Kulbachinskii, V. G. Kytin, Z. V. Lavrukhina, A. N. Kuznetsov, and A. V. Shevelkov, Galvanomagnetic and thermoelectric properties of $\mathrm{BiTeBr}$ and BiTeI single crystals and their electronic structure, Semiconductors 44, 1548 (2010).

[40] I. A. Nechaev and E. V. Chulkov, Width of the quasiparticle spectral function in a two-dimensional electron gas with spin-orbit interaction, Phys. Solid State 51, 1772 (2009). 
[41] I. A. Nechaev, P. M. Echenique, and E. V. Chulkov, Inelastic decay rate of quasiparticles in a two-dimensional spin-orbit coupled electron system, Phys. Rev. B 81, 195112 (2010).

[42] I. A. Nechaev, V. M. Silkin, and E. V. Chulkov, Features of quasiparticle decay in $2 \mathrm{~d}$ electronic systems with spin-orbit interaction, J. Exp. Theor. Phys. 112, 134 (2011).
[43] B. Diaconescu, K. Pohl, L. Vattuone, L. Savio, P. Hofmann, V. M. Silkin, J. M. Pitarke, E. V. Chulkov, P. M. Echenique, D. Farías, and M. Rocca, Low-energy acoustic plasmons at metal surfaces, Nature (London) 448, 57 (2007).

[44] F. Stern, Polarizability of a Two-Dimensional Electron Gas, Phys. Rev. Lett. 18, 546 (1967). 